\title{
Circulating level of microRNA-142-5p is a potential biomarker for predicting in-stent restenosis: a case-control study
}

\author{
Chun-Hsu Pan ${ }^{1,2 \dagger}$, Shu-Chen Chien ${ }^{2,3 \dagger}{ }^{\text {, Chang-Jui Chen }}{ }^{2,3}$, Chun-Ming Shih ${ }^{4}$, Ming-Hsiung Hsieh ${ }^{5}$, \\ Chun-Yao Huang ${ }^{4}$, Wei-Fung Bi ${ }^{4}$, Chao-Shun Chan ${ }^{4}$, Yung-Ta Kao ${ }^{4}$, Cheng-Yi Hsiao ${ }^{4}$, Shuo-Ju Chiang ${ }^{4}$, \\ Kuang-Hsing Chiang ${ }^{4}$, Jen-Hung Huang ${ }^{5}$, Yun-Ru Liu ${ }^{6}$, Ji-Dung Luo ${ }^{7}$, Hui-Yu Huang ${ }^{8}$ and Chieh-Hsi Wu2 ${ }^{*}$
}

\begin{abstract}
Background: Patients who receive percutaneous coronary intervention (PCI) have different chances of developing in-stent restenosis (ISR). To date, no predictable biomarker can be applied in the clinic. MicroRNAs (miRNAs or miRs) play critical roles in transcription regulation, and their circulating levels were reported to have potential as clinical biomarkers.

Methods: In total, 93 coronary stent-implanted patients without pregnancy, liver or renal dysfunction, malignancy, hemophilia, or autoimmune diseases were recruited in this clinical study. All recruited participants were divided into an ISR group $(n=45)$ and a non-ISR group $(n=48)$ based on their restenotic status as confirmed by cardiologists at the first follow-up visit ( 6 months after surgery). Blood samples of all participants were harvested to measure circulating levels of miRNA candidates (miR-132, miR-142-5p, miR-15b, miR-24-2, and miR-424) to evaluate whether these circulating miRNAs can be applied as predictive biomarkers of ISR.

Results: Our data indicated that circulating levels of miR-142-5p were significantly higher in the ISR population, and results from the receiver operating characteristic $(R O C)$ curve analysis also demonstrated superior discriminatory ability of miR-142-5p in predicting patients' restenotic status. In addition, circulating levels of miR-15b, miR-24-2, and miR-424 had differential expressions in participants with diabetes, hyperlipidemia, and hypertension, respectively.
\end{abstract}

Conclusions: The current study revealed that the circulating level of miR-142-5p has potential application as a clinical biomarker for predicting the development of ISR in stent-implanted patients.

Keywords: Biomarker, Circulating level, In-stent restenosis, miRNA

\section{Background}

Restenosis after a percutaneous coronary intervention (PCI) has historically been described as an "Achilles heel" [1]. Although the invention of drug-eluting stents (DESs) improved the restenosis incidence rate to $10-15 \%$, compared to the $25-30 \%$ rate of bare-metal stents (BMSs),

\footnotetext{
*Correspondence: chhswu@tmu.edu.tw

†Equal contribution as first authors: Chun-Hsu Pan and Shu-Chen Chien

2 School of Pharmacy, Taipei Medical University, Taipei 11031, Taiwan

Full list of author information is available at the end of the article
}

restenosis remains a clinical challenge in the real world [2]. Despite a higher restenosis rate with BMSs than with DESs, the much-higher cost of DESs and uncertain safety and efficacy are factors favoring BMS use [3]. Numerous studies have investigated the question of whether DESs outperform BMSs. Piccolo and colleagues recently conducted a meta-analysis of 20 randomized control trials to compare the safety and efficacy performance between new-generation DESs and BMSs [4]. This elicited the question as to whether the BMS option is still needed in current practice. Colombo and colleagues addressed 
this issue and remarked that BMS management should remain in current practice, especially for patients who cannot complete dual antiplatelet therapy, patients with high-bleeding risks, and patients who need non-cardiac surgery [5]. Therefore, the best strategy is to categorize patients and thus optimize personalized management when choosing a stent.

MicroRNA (miRNAs or miRs) are small noncoding RNAs (with ca. 21-25 nucleotides) that play vital roles in post-transcription of specific messenger (m)RNA targets [6]. Studies demonstrated that miRNAs regulate nearly all physiological processes and are associated with several human diseases, including cancer, angiogenesis, immune-related diseases, and neurodegenerative diseases [7-10]. In fact, miRNAs are recognized as disease indicators, because expressions of distinct miRNAs may indicate specific cancer types. Also, miRNAs with characteristic roles reflect different hematopoietic lineages [11]. In addition, noninvasive and sensitive features of miRNAs have attracted attention due to their potential as a tactic in tackling this "Achilles heel" challenge [12]. As a result, miRNAs could be the best fit to investigate the complex restenosis pathology with their disease-specific and ideal-biomarker characteristics. Several miRNAs, including miR-132, miR-142-5p, miR-15b, miR-24-2, and miR-424, were reported to modulate cellular behaviors or functions in vascular smooth muscle cells and endothelial cells as well as to regulate restenotic progression in animal studies [13-21]. The objective of this study was to clarify correlations of circulating levels of five miRNAs and the prevalence of in-stent restenosis (ISR) in patients who had received vascular stent implantation.

\section{Methods}

\section{Clinical study}

This study was approved by the Taipei Medical University Joint Institutional Review Board (TMU-JIRB No. 201405019), and all experiment protocols were in accordance with the Declaration of Helsinki. All participants signed informed consent forms and were recruited by cardiologists at Taipei Medical University Hospital and Taipei Municipal Wanfang Hospital from June 2015 to May 2016. The inclusion criterion was patients who had undergone PCI, and exclusion criteria were patients who were pregnant, and those who had been diagnosed with liver diseases (liver dysfunction), kidney dysfunction (dialysis), malignancy, hemophilia, or autoimmune diseases. In total, 93 patients were recruited and analyzed. Participants were divided into two groups (ISR and nonISR) based on their restenotic status as confirmed by cardiologists at the first follow-up visit (6 months after surgery). The ISR group was defined as having a greater than $50 \%$ decrease in the luminal diameter. Patients were enrolled by cardiologists and needed to have $15 \mathrm{~mL}$ of blood taken for analysis. Blood was obtained by a certificated medical technologist or nurse in each medical facility. Blood was collected in anticoagulant tubes and was stored at $2-8{ }^{\circ} \mathrm{C}$ until further processing.

\section{Purification and reverse transcription (RT) of miRNA}

Serum miRNA was purified using a miRNeasy Serum/ Plasma Mini Kit (\#217004, Qiagen, Venlo, the Netherlands) according to the original manufacturer's protocol. The RT reaction of miRNA for each sample was performed using a miScript II RT kit (\#218161, Qiagen) to obtain complementary (c)DNA based on the manufacturer's instructions.

\section{Quantitative polymerase chain reaction (qPCR)}

Serum miRNA expression was measured in plasma of each patient using a qPCR. U6 non-coding small nuclear (U6 sn)RNA was employed as an interval control. Primers were synthesized by Qiagen, and primer sequences are presented in Table 1. The miScript SYBR Green PCR kit (\#218073, Qiagen) and miScript Primer Assay (\#218300, Qiagen) were used with the $\mathrm{CFX}^{\mathrm{TM}}{ }^{\mathrm{TM}}$ RealTime PCR Detection System (Bio-Rad, Hercules, CA, USA) to enable quantification of mature miRNA. Cycling conditions for the PCR were as follows: initial activation at $95{ }^{\circ} \mathrm{C}$ for $15 \mathrm{~min}$, followed by 40 cycles of three-step cycling including denaturation at $94{ }^{\circ} \mathrm{C}$ for $15 \mathrm{~s}$, annealing at $55{ }^{\circ} \mathrm{C}$ for $30 \mathrm{~s}$, and extension at $70{ }^{\circ} \mathrm{C}$ for $30 \mathrm{~s}$, at which time, fluorescence data were collected. This procedure was repeated three times for each sample with a total reaction volume of $25 \mu \mathrm{L}$. The relative transcript expression was calculated using the equation. $2^{-\Delta \Delta C t}$.

\section{Statistical analysis}

All values are presented as the mean \pm standard deviation (SD). Differences among multiple groups were determined using a one-way analysis of variance (ANOVA)

Table 1 Sequences of primers used for detecting micro (mi)RNAs

\begin{tabular}{lll}
\hline Target gene & miRBase accession & Primer sequences \\
\hline miR-132 & MIMAT0000426 & UAACAGUCUACAGCCAUGGUCG \\
miR-142-5p & MIMAT0000433 & CAUAAAGUAGAAAGCACUACU \\
miR-15b & MIMAT0000417 & UAGCAGCACAUCAUGGUUUACA \\
miR-24-2 & MIMAT0004497 & UGCCUACUGAGCUGAAACACAG \\
miR-424 & MIMAT0001341 & CAGCAGCAAUUCAUGUUUUGAA \\
U6 snRNA & - & CTCGCTTCGGCAGCACA \\
& & AACGCTTCACGAATTGCGT
\end{tabular}

* The miScript Universal Primer (Qiagen) was used as a reverse primer for detecting mRNAs 
in combination with Dunnett's test. A value of $p<0.05$ was considered statistically significant. Statistical assays were performed using SAS software (vers. 9.4; SAS Institute, Cary, NC, USA). A receiver operating characteristic (ROC) curve was drawn to assess the predictive accuracy of the biomarkers. We evaluated the discriminative power of the biomarker candidates by calculating the area under the ROC curve (AUC). A diagnostic test with an AUC of 1.0 was defined as a perfect diagnostic test, whereas an AUC of 0.5 was regarded as a non-discriminating test. Chi-squared test was used to compare correlations of different variables between the restenosis and non-restenosis groups. A logistic regression analysis was performed by a previously described method [22]

\section{Results}

\section{Patient characteristics}

Basic demographic data of recruited patients are shown in Table 2. The recruiting age range was $30-80$ years. In the present study, 93 patients were analyzed (including 45 patients in the ISR group and 48 patients in the nonISR group). Age, clinical comorbidities, and medications were comparable between the ISR and non-ISR groups. Apart from clopidogrel, which significantly differed,

Table 2 Clinical demographics of the ISR and non-ISR groups in this study

\begin{tabular}{|c|c|c|c|c|}
\hline Parameter & $\begin{array}{l}\text { Total } \\
(N=93)\end{array}$ & $\begin{array}{l}\text { ISR } \\
(N=45)\end{array}$ & $\begin{array}{l}\text { Non-ISR } \\
(N=48)\end{array}$ & $p$ value \\
\hline Age (years) & $65.71 \pm 8.73$ & $65.69 \pm 8.82$ & $65.61 \pm 8.72$ & 0.12 \\
\hline \multicolumn{5}{|l|}{ Gender } \\
\hline Male & $76(81.72 \%)$ & 41 (91.11\%) & 35 (72.92\%) & - \\
\hline Female & $17(18.28 \%)$ & 4 (8.89\%) & $13(27.08 \%)$ & - \\
\hline Smoking & $3(3.23 \%)$ & $2(4.44 \%)$ & $1(2.08 \%)$ & - \\
\hline \multicolumn{5}{|l|}{ Comorbidities } \\
\hline Hyperlipidemia & 84 (90.32\%) & 42 (93.33\%) & $42(87.50 \%)$ & 0.34 \\
\hline Hypertension & 71 (76.34\%) & 33 (73.33\%) & 38 (79.17\%) & 0.51 \\
\hline Angina pectoris & $42(45.16 \%)$ & $22(48.89 \%)$ & $20(41.67 \%)$ & - \\
\hline Diabetes & $29(31.18 \%)$ & $15(33.33 \%)$ & $14(29.17 \%)$ & 0.67 \\
\hline Chronic CHF & $5(5.38 \%)$ & $3(6.67 \%)$ & $2(4.17 \%)$ & - \\
\hline \multicolumn{5}{|l|}{ Medications } \\
\hline Aspirin & 75 (80.65\%) & 40 (88.89\%) & 35 (72.92\%) & 0.05 \\
\hline Beta blocker & $51(54.84 \%)$ & $29(64.44 \%)$ & $22(45.83 \%)$ & 0.07 \\
\hline CCBs & $30(32.26 \%)$ & $14(31.11 \%)$ & $16(33.33 \%)$ & 0.82 \\
\hline ARB & $61(65.59 \%)$ & $30(66.67 \%)$ & $31(64.58 \%)$ & 0.83 \\
\hline Clopidogrel & $43(46.24 \%)$ & 15 (33.33\%) & $28(58.33 \%)$ & 0.02 \\
\hline $\begin{array}{l}\text { Lipid-lowering } \\
\text { agent }\end{array}$ & 84 (90.32\%) & 41 (91.11\%) & 43 (89.58\%) & 0.80 \\
\hline
\end{tabular}

Data are presented as the mean \pm standard deviation or number (percentage). $\mathrm{CHF}$, cardiac heart failure; $C \mathrm{CBs}$, calcium channel blockers; $\mathrm{ARB}$, angiotensin II receptor blocker there were no other significant differences among clinical characteristics between the ISR and non-ISR groups.

\section{Circulating levels of miRNA candidates}

To compare the difference in expressions of each miRNA, we performed a qPCR to measure relative levels of circulating miRNAs of the ISR group compared to the nonISR group. We observed that two miRNAs (miR-132 and miR-24-2) were lower, while the other miRNAs (miR15b, miR-424, and miR-142-5p) were higher in the ISR population. Previous studies proposed that miR-132 and miR-24-2 might have a protective function against restenotic processes $[13,14,17,18]$. On the other hand, miR$142-5 p$ showed a significantly higher abundance in the restenosis population over the non-restenosis population. This observation of miR-142-5p expression levels is consistent with our hypothesis, according to a literature review. That is, miR-142-5p may increase the restenosis risk. Interestingly, miR-15b and miR-424 appeared to be slightly higher in the restenosis population compared to the non-restenosis group. Previous studies regarding miR-15b and miR-424 demonstrated their protective and preventive potentials against atherosclerosis development in a murine model, but opposite effects were shown in this human population (Fig. 1).

\section{ROC performances of miRNA biomarkers and comparisons} in human plasma

To test these miRNA roles as clinical prognostic biomarkers, we conducted a ROC analysis and assessed the diagnostic value of each of the five selected miRNAs. Detailed information from the ROC analysis as to the ability of the five miRNAs to diagnose restenosis in patients is shown in Table 3. Consistent with expression level results, miR142-5p showed the highest predictive value (with an AUC of 0.734), which indicated good diagnostic accuracy [23]. The AUC of miR-424 (0.599) was second behind miR-142-5p, and was on the edge of exhibiting sufficient diagnostic accuracy. The AUC results for miR-15b, miR132, and miR-24-2 fell in the range considered to have no diagnostic value despite their high specificity for restenosis (at 0.466, 0.425, and 0.364 respectively, with an AUC of $<0.5$ considered to have no diagnostic value). These results demonstrated that miR-142-5p had a superior diagnostic performance among the five miRNAs for discriminating patients with from those without a high risk of restenosis.

\section{Effects of comorbidities in circulating levels of miRNA candidates of participants}

Hyperlipidemia, hypertension, angina pectoris, and diabetes are common clinical comorbidities in patients treated with percutaneous transluminal coronary 


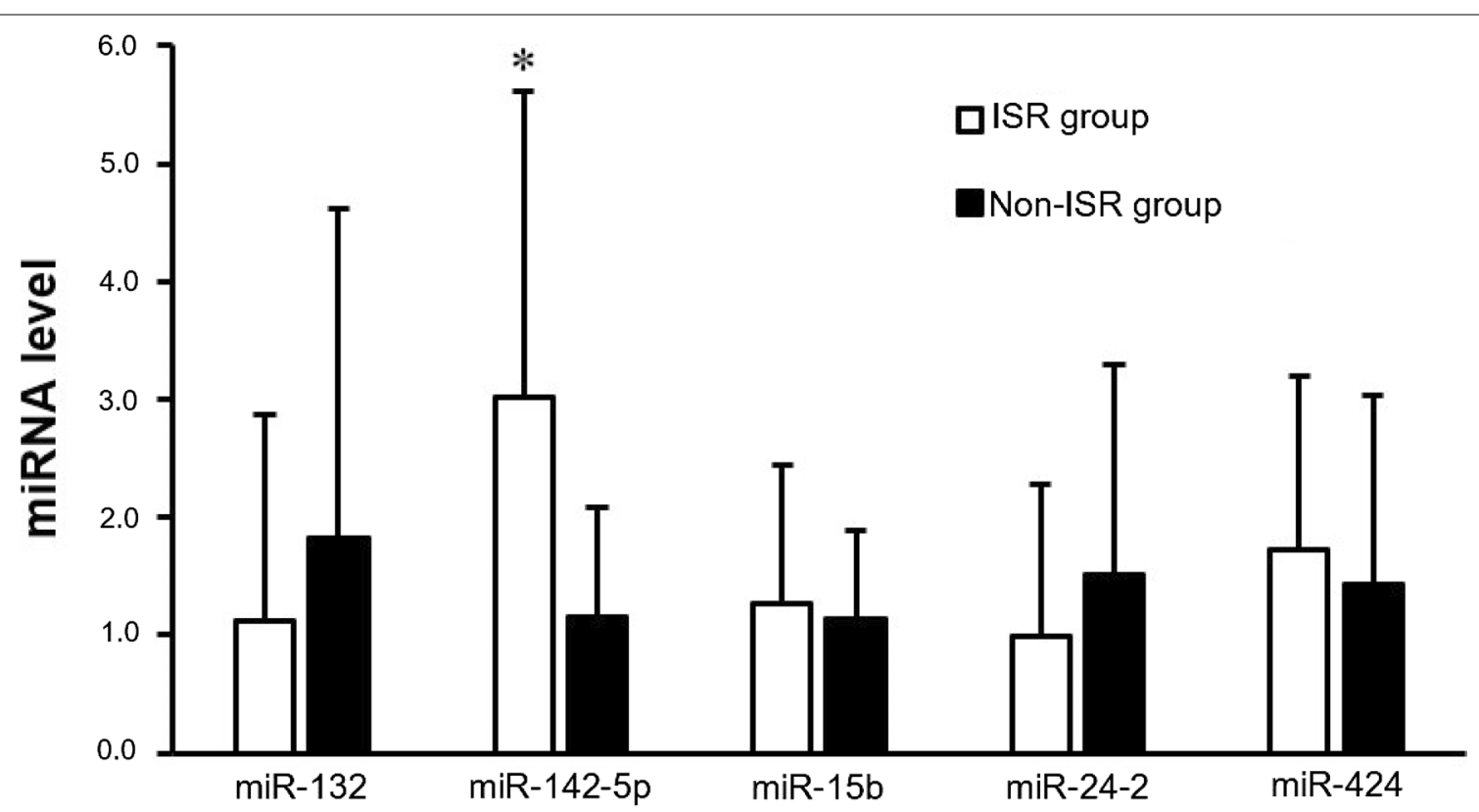

Fig. 1 Circulating levels of miRNAs in the ISR and non-ISR populations. ${ }^{*} p<0.05$, indicates a significant difference between the two groups

Table 3 Receiver operating characteristic (ROC) curve analysis of miRNAs between the ISR and non-ISR populations

\begin{tabular}{lllllll}
\hline miRNA & AUC & $\mathbf{9 5 \%} \mathbf{C l}$ & $\boldsymbol{p}$ & Sensitivity & Specificity & Accuracy \\
\hline miR-132 & 0.425 & {$[-0.209,0.059]$} & 0.274 & 0.059 & 0.973 & 0.535 \\
miR-142-5p & 0.734 & {$[0.093,0.375]$} & 0.001 & 0.432 & 1.000 & 0.571 \\
miR-15b & 0.466 & {$[-0.061,0.094]$} & 0.606 & 0.086 & 0.821 & 0.543 \\
miR-24-2 & 0.364 & {$[-0.268,-0.003]$} & 0.045 & 0.059 & 0.943 & 0.507 \\
miR-424 & 0.599 & {$[-0.027,0.226]$} & 0.124 & 0.692 & 0.575 & 0.633 \\
\hline
\end{tabular}

$\mathrm{AUC}$, are under the ROC curve; $\mathrm{Cl}$, confidence interval

angioplasty (PTCA). It was unclear whether these comorbidities might influence circulating levels of our examined miRNA candidates between the ISR and nonISR groups. Experimental results showed that circulating miR-142-5p and miR-24-2 significantly differed between the ISR and non-ISR groups in participants with hyperlipidemia (Table 4). Similarly, markedly higher miR-142-5p and miR-424 levels were found in the ISR group in participants with hypertension (Table 5). In participants with diabetes, plasma levels of miR-132 and miR-424 were significantly lower in the ISR group compared to those in the non-ISR group (Table 6). However, angina pectoris had no impact on circulating levels of any of these miRNA candidates between the ISR and non-ISR groups (Table 7).
Table 4 Comparison of miRNA levels between the ISR and non-ISR groups in participants with hyperlipidemia $(N=84)$

\begin{tabular}{llll}
\hline miRNA & Group & \\
\cline { 2 - 4 } & ISR & Non-ISR & $\boldsymbol{p}$ value \\
\hline miR-132 & $1.055 \pm 1.750$ & $1.963 \pm 2.908$ & 0.074 \\
miR-142-5p & $3.047 \pm 2.521$ & $1.186 \pm 0.971$ & $0.019^{\mathrm{a}}$ \\
miR-15b & $1.217 \pm 1.153$ & $1.086 \pm 0.676$ & 0.519 \\
miR-24-2 & $0.835 \pm 0.934$ & $1.607 \pm 1.884$ & $0.022^{\mathrm{a}}$ \\
miR-424 & $1.664 \pm 1.531$ & $1.544 \pm 1.646$ & 0.450
\end{tabular}

a Indicates a statistical difference between the two groups

Data are presented as the mean \pm standard deviation

\section{Discussion}

ISR refers to a reduction in coronary vessel diameter 
Table 5 Comparison of miRNA levels between the ISR and non-ISR groups in participants with hypertension $(N=71)$

\begin{tabular}{llll}
\hline miRNA & Group & \\
\cline { 2 - 4 } & ISR & Non-ISR & $\boldsymbol{p}$ value \\
\hline miR-132 & $1.313 \pm 2.005$ & $1.629 \pm 2.439$ & 0.587 \\
miR-142-5p & $2.817 \pm 1.928$ & $1.297 \pm 1.010$ & $0.026^{\mathrm{a}}$ \\
miR-15b & $1.284 \pm 1.195$ & $1.111 \pm 0.693$ & 0.956 \\
miR-24-2 & $0.919 \pm 0.990$ & $1.528 \pm 1.967$ & 0.281 \\
miR-424 & $2.058 \pm 1.620$ & $1.287 \pm 1.066$ & $0.028^{\mathrm{a}}$ \\
\hline
\end{tabular}

a Indicates a statistically significant difference between the two groups Data are presented as the mean \pm standard deviation

Table 6 Comparison of miRNA levels between the ISR and non-ISR groups in participants with diabetes $(N=29)$

\begin{tabular}{llll}
\hline miRNA & Group & & \\
\cline { 2 - 4 } & ISR & Non-ISR & $\boldsymbol{p}$ value \\
\hline miR-132 & $1.530 \pm 2.276$ & $3.664 \pm 4.368$ & $0.047^{\mathrm{a}}$ \\
miR-142-5p & $3.307 \pm 2.578$ & $1.233 \pm 1.358$ & 0.097 \\
miR-15b & $1.213 \pm 1.137$ & $1.767 \pm 0.971$ & $0.033^{\mathrm{a}}$ \\
miR-24-2 & $1.613 \pm 2.023$ & $2.558 \pm 2.805$ & 0.412 \\
miR-424 & $1.778 \pm 1.594$ & $2.007 \pm 2.432$ & 0.801 \\
\hline
\end{tabular}

${ }^{a}$ Indicates a statistically significant difference between the two groups Data are presented as the mean \pm standard deviation

Table 7 Comparison of miRNA levels between the ISR and non-ISR groups in participants with angina pectoris $(N=31)$

\begin{tabular}{llll}
\hline miRNA & Group & \\
\cline { 2 - 4 } & ISR & Non-ISR & $\boldsymbol{p}$ value \\
\hline miR-132 & $0.563 \pm 0.394$ & $1.221 \pm 1.350$ & 0.277 \\
miR-142-5p & $2.195 \pm 1.409$ & $1.496 \pm 1.368$ & 0.637 \\
miR-15b & $0.797 \pm 0.529$ & $0.819 \pm 0.314$ & 0.563 \\
miR-24-2 & $0.498 \pm 0.314$ & $1.118 \pm 0.763$ & 0.060 \\
miR-424 & $1.590 \pm 1.264$ & $1.227 \pm 0.57$ & 0.392 \\
\hline
\end{tabular}

Data are presented as the mean \pm standard deviation

after a PCI, which remains a major clinical problem in the cardiovascular field. The pathological processes of restenosis are known to be complicated due to the many factors involved [24]. Some biological and genetic factors, such as drug resistance [25], systemic and allergic inflammation [26, 27], neoatherosclerosis [28], variants of the $\beta 2$-adrenergic receptor [29], and polymorphisms of the tumor necrosis factor- $\alpha$ gene [30], were reported to participate in the progression of DES restenosis.
Subclinical hypothyroidism and inflammatory activity were also demonstrated to regulate the stability of atherosclerotic plaques isolated from internal carotid artery stenosis [31]. Diabetes is a risk factor which strikingly increases the prevalence of, incidence of, and mortality due to cardiovascular diseases [32]. ISR was confirmed to be positively associated with mean blood glucose levels, and strict peri-procedural glycemic control could improve outcomes after a PCI in patients with an acute ST-elevation myocardial infarction [33]. Pathophysiological activation of the ubiquitin proteasome system was shown to be involved in several pathological characteristics (e.g., endothelial dysfunction and plaque destabilization) during atherosclerosis progression in diabetic patients and to be one of the mechanisms causing the generation of insulin resistance [34]. Moreover, the prospective AIRE Study indicated that lower levels of serum adiponectin, an adipocyte-derived factor with an antidiabetic effect, were related to restenosis even in subjects with normal glucose tolerance [35]. Epigenetic markers are reversible, which can reprogram genetic dysregulation independent of inherited DNA sequences. Therefore, epigenetic merits are applied to prognostic biomarkers and even therapeutic targets in various fields. miRNAs are one kind of emerging epigenetic marker in advancing cardiovascular research during the last decade [36, 37]. However, molecular mechanisms linking epigenetic and genetic phenomena are still lacking. Moreover, miRNA biomarker research in restenosis remains limited. The present study attempted to investigate potential miRNA biomarkers to characterize restenosis risk levels among patients who had undergone PCI management by analyzing genetics to further explore molecular mechanisms.

Although several miRNAs have been studied and found to be involved in angiogenesis or vascular neointima, our findings in human clinical samples highlight crucial aspects that have, to our knowledge, not been underscored before. First, Xu et al. argued that miR-15b modulates the smooth muscle cell phenotype through targeting the yes-associated protein (YAP) in a rat model, which may decrease the restenosis risk [38]. Our data demonstrated an opposite phenomenon when translated from a rat model to human samples. Costa de Freitas et al. proved that clopidogrel exposure modulates miR15b-5p and miR-26a-5p expression levels in vitro [39]. In our study, $46.24 \%$ of participants were using clopidogrel, and the data showed a significant difference between the restenosis and non-restenosis populations. As opposed to the animal model, clinical human-environmental influences are much more complicated. Medication administration serves an essential role in regulating epigenetic marker performance. Second, Muñoz-Pacheco et al. indicated that ezetimibe suppresses the extracellular 
signal-regulated kinase (ERK)/mitogen-activated protein kinase (MAPK) pathway and nuclear factor (NF)- $\mathrm{kB}$ activity and thus downregulates miR-424 expression. Those findings suggest that ezetimibe's anti-atherosclerotic effects occur through inhibiting miRNAs [40]. Around $90 \%$ of our participants were using lipid-lowering agents. That may explain the discrepancy in the hypothetical role of miR-424 in restenosis prognosis. Interestingly, Richardsen et al. recently argued that low expression of miR-424 was correlated with an aggressive prostate cancer phenotype, and thus may worsen clinical outcomes and failure. Richardsen et al. concluded that the roles of miR-424 in regulating host immune responses among different cancer types remain to be further elucidated [41]. Not only medical use, but also the disease state and type are also critical points determining miRNA epigenetic marker regulation. Last, miRNAs have been characterized as ideal biomarkers due to their specific, sensitive, predictive, and non-invasive features [42, 43]. The ROC analysis of these five miRNAs presented their potential performance in stratifying risk levels for restenosis.

Some limitations of this study might be of concern. First, the sample size of recruited patients was small, and it might not have sufficient power to fully clarify relationships between circulating levels of miRNA candidates and the restenotic status under different conditions (e.g., comorbidities, age, gender, medicinal interventions, diet, or lifestyle). Second, this project was not a clinical study that recruited multiple racial participants; thus these data might not be applicable to different ethnic groups. Finally, long-term follow-up with multiple observation times are needed to clarify whether correlations between circulating miRNAs and the restenotic status change over time.

\section{Conclusions}

Personalized medicine has potential benefits to substantially improve outcomes of clinical interventions by the tailored therapies based on individual patient's characteristics, such as genetic information, physiological status, diet and lifestyle. Predictable biomarkers of ISR could be clinically applied for reducing wasteful practices by helping us to distinguish precisely who belongs to the susceptible population and then the most suitable intervention (e.g., bare-metal stent or drug-eluting stent implantation) could be chosen for individual patients. In this study, we established an analysis of dynamic molecular mechanisms through epigenetic human clinical trials of complicated restenosis pathological processes. The data indicated that miR-142-5p has significant potential to predict the population at high risk of restenosis, while miR-132 and miR-24-2 may be beneficial in decreasing the restenosis risk. These miRNA epigenetic markers demonstrated the real-time and dynamic state of individuals with their comorbidities and medication use. Our study offers potential biomarker tools and possible molecular mechanisms for targeting in-stent restenosis.

\section{Acknowledgements \\ We thank the TMU Office of Research and Development for improving the manuscript through their editing service.}

\section{Authors' contributions}

Chien SC, Shih CM, Huang HY, and Wu CH provided conception and design of the experiments. Chen CJ, Hsieh MH, Huang CY, Pan CH, Bi WF, Chan CS, Kao YT, Hsiao CY, Chiang SJ, Chiang KH, Huang JH, Liu YR, and Luo JD performed the experiments, data analysis, and interpretation of the data. The manuscript was written by Chen CJ, Pan CH, Huang HY, and Wu CH. Wu CH contributed to funding acquisition. All authors read and approved the final version for manuscript

\section{Funding}

This work was supported by Taipei Medical University Hospital (104TMUTMUH-17), China Medical University (CMU97-079), and the Ministry of Science and Technology of Taiwan (MOST 105-2320-B-038-042, MOST 107-2320-B-038028, and MOST 108-2320-B-038-040-MY3).

\section{Availability of data and materials}

The data used to support the findings of this study are available from the corresponding author upon reasonable request.

\section{Consent for publication}

Not applicable.

\section{Ethics approval and consent to participate}

The Taipei Medical University Joint Institutional Review Board approved this study (TMU-JIRB No. 201405019), and informed written consent was obtained from all participants.

\section{Competing interest}

The authors declare that they have no conflicts of interest.

\section{Author details}

${ }^{1}$ PhD Program in Drug Discovery and Development Industry, College of Pharmacy, Taipei Medical University, Taipei 11031, Taiwan. ${ }^{2}$ School of Pharmacy, Taipei Medical University, Taipei 11031, Taiwan. ${ }^{3}$ Department of Pharmacy, Taipei Medical University Hospital, Taipei 11031, Taiwan. ${ }^{4}$ Department of Cardiology, Taipei Medical University Hospital, Taipei 11031, Taiwan. ${ }^{5}$ Department of Cardiology, Taipei Municipal Wanfang Hospital, Taipei Medical University, Taipei 11031, Taiwan. ${ }^{6}$ Joint Biobank, Office of Human Research, Taipei Medical University, Taipei 11031, Taiwan. ${ }^{7}$ Bioinformatics Resource Center, The Rockefeller University, New York, NY 10065, USA. ${ }^{8}$ Graduate Institute of Metabolism and Obesity Sciences, Taipei Medical University, Taipei 11031, Taiwan.

Received: 28 November 2020 Accepted: 19 January 2021

Published online: 08 February 2021

\section{References}

1. Dangas G, Fuster V. Management of restenosis after coronary intervention. Am Heart J. 1996;132:428-36.

2. Looser PM, Kim LK, Feldman DN. In-stent restenosis: pathophysiology and treatment. Curr Treat Options Cardiovasc Med. 2016;18:10.

3. Alfonso F, Byrne RA, Rivero F, Kastrati A. Current treatment of in-stent restenosis. J Am Coll Cardiol. 2014;63:2659-73.

4. Piccolo R, Bonaa KH, Efthimiou O, Varenne O, Baldo A, Urban P, Kaiser C, Remkes W, Raber L, de Belder A, van 't Hof AWJ, Stankovic G, Lemos PA, Wilsgaard T, Reifart J, Rodriguez AE, Ribeiro EE, Serruys P, Abizaid A, Sabate M, Byrne RA, de la Torre Hernandez JM, Wijns W, Juni P, Windecker S, Valgimigli M, Coronary Stent Trialists C. Drug-eluting or bare-metal stents for percutaneous coronary intervention: a systematic review and 
individual patient data meta-analysis of randomised clinical trials. Lancet. 2019;393:2503-10.

5. Colombo A, Giannini F, Briguori C. Should we still have bare-metal stents available in our catheterization laboratory? J Am Coll Cardiol. 2017;70:607-19.

6. Wahid F, Shehzad A, Khan T, Kim YY. MicroRNAs: synthesis, mechanism, function, and recent clinical trials. Biochim Biophys Acta. 2010;1803:1231-43.

7. Oliveto S, Mancino M, Manfrini N, Biffo S. Role of microRNAs in translation regulation and cancer. World J Biol Chem. 2017:8:45-56.

8. Raoof R, Jimenez-Mateos EM, Bauer S, Tackenberg B, Rosenow F, Lang J, Onugoren MD, Hamer H, Huchtemann T, Kortvelyessy P, Connolly NMC, Pfeiffer S, Prehn JHM, Farrell MA, O'Brien DF, Henshall DC, Mooney C. Cerebrospinal fluid microRNAs are potential biomarkers of temporal lobe epilepsy and status epilepticus. Sci Rep. 2017;7:3328.

9. Tiwari D, Peariso K, Gross C. MicroRNA-induced silencing in epilepsy: opportunities and challenges for clinical application. Dev Dyn. 2018;247:94-110.

10. Prabahar A, Natarajan J. Prediction of microRNAs involved in immune system diseases through network based features. J Biomed Inform. 2017;65:34-45.

11. Chung SS, Hu W, Park CY. The role of MicroRNAs in hematopoietic stem cell and leukemic stem cell function. Ther Adv Hematol. 2011;2:317-34.

12. Etheridge A, Lee I, Hood L, Galas D, Wang K. Extracellular microRNA: a new source of biomarkers. Mutat Res. 2011;717:85-90.

13. Choe N, Kwon JS, Kim JR, Eom GH, Kim Y, Nam Kl, Ahn Y, Kee HJ, Kook H. The microRNA miR-132 targets Lrrfip1 to block vascular smooth muscle cell proliferation and neointimal hyperplasia. Atherosclerosis. 2013;229:348-55.

14. Wang W, Li X, Ren L, Yuan C, Han Y, Wang Z. MiR-132 relieves vascular endothelial inflammation and improve endothelial function in atherosclerosis rats by regulating SIRT1. Minerva Endocrinol. 2019.

15. Xu R, Bi C, Song J, Wang L, Ge C, Liu X, Zhang M. Upregulation of miR-142-5p in atherosclerotic plaques and regulation of oxidized low-density lipoprotein-induced apoptosis in macrophages. Mol Med Rep. 2015;11:3229-34.

16. Ma Z, Liu T, Huang W, Liu H, Zhang HM, Li Q, Chen Z, Guo AY. MicroRNA regulatory pathway analysis identifies miR-142-5p as a negative regulator of TGF-beta pathway via targeting SMAD3. Oncotarget. 2016;7:71504-13.

17. Hernandez-Torres F, Aranega AE, Franco D. Identification of regulatory elements directing miR-23a-miR-27a-miR-24-2 transcriptional regulation in response to muscle hypertrophic stimuli. Biochim Biophys Acta. 2014;1839:885-97.

18. Lee SH, Chen TY, Dhar SS, Gu B, Chen K, Kim YZ, Li W, Lee MG. A feedback loop comprising PRMT7 and miR-24-2 interplays with Oct4, Nanog, Klf4 and c-Myc to regulate stemness. Nucleic Acids Res. 2016;44:10603-18.

19. Merlet E, Atassi F, Motiani RK, Mougenot N, Jacquet A, Nadaud S, Capiod T, Trebak M, Lompre AM, Marchand A. miR-424/322 regulates vascular smooth muscle cell phenotype and neointimal formation in the rat. Cardiovasc Res. 2013:98:458-68

20. Tsai SH, Huang PH, Tsai HY, Hsu YJ, Chen YW, Wang JC, Chen YH, Lin SJ. Roles of the hypoximir microRNA-424/322 in acute hypoxia and hypoxia-induced pulmonary vascular leakage. FASEB J. 2019;33:12565-75.

21. Chamorro-Jorganes A, Araldi E, Penalva LO, Sandhu D, Fernandez-Hernando C, Suarez Y. MicroRNA-16 and microRNA-424 regulate cell-autonomous angiogenic functions in endothelial cells via targeting vascular endothelial growth factor receptor-2 and fibroblast growth factor receptor-1. Arterioscler Thromb Vasc Biol. 2011;31:2595-606.

22. Lehmann S, Dumurgier J, Schraen S, Wallon D, Blanc F, Magnin E, Bombois S, Bousiges O, Campion D, Cretin B, Delaby C, Hannequin D, Jung B, Hugon J, Laplanche JL, Miguet-Alfonsi C, Peoc'h K, Philippi N, Quillard-Muraine M, Sablonniere B, Touchon J, Vercruysse O, Paquet C, Pasquier F, Gabelle A. A diagnostic scale for Alzheimer's disease based on cerebrospinal fluid biomarker profiles. Alzheimers Res Ther. 2014;6:38.

23. Simundic AM. Measures of diagnostic accuracy: basic definitions. EJIFCC. 2009;19:203-11.

24. Aoki J, Tanabe K. Mechanisms of drug-eluting stent restenosis. Cardiovasc Interv Ther. 2020.

25. Huang S, Houghton PJ. Mechanisms of resistance to rapamycins. Drug Resist Updat. 2001:4:378-91.

26. Niccoli G, Montone RA, Ferrante G, Crea F. The evolving role of inflammatory biomarkers in risk assessment after stent implantation. J Am Coll Cardiol. 2010;56:1783-93.

27. Koster R, Vieluf D, Kiehn M, Sommerauer M, Kahler J, Baldus S, Meinertz T, Hamm CW. Nickel and molybdenum contact allergies in patients with coronary in-stent restenosis. Lancet. 2000;356:1895-7.
28. Park SJ, Kang SJ, Virmani R, Nakano M, Ueda Y. In-stent neoatherosclerosis: a final common pathway of late stent failure. J Am Coll Cardiol. 2012;59:2051-7.

29. Monraats PS, Pires NM, Agema WR, Zwinderman AH, Schepers A, de Maat MP, Doevendans PA, de Winter RJ, Tio RA, Waltenberger J, Frants RR, Quax $\mathrm{PH}$, van Vlijmen BJ, Atsma DE, van der Laarse A, van der Wall EE, Jukema JW. Genetic inflammatory factors predict restenosis after percutaneous coronary interventions. Circulation. 2005;112:2417-25.

30. Monraats PS, Pires NM, Schepers A, Agema WR, Boesten LS, de Vries MR, Zwinderman AH, de Maat MP, Doevendans PA, de Winter RJ, Tio RA, Waltenberger J, t Hart LM, Frants RR, Quax PH, van Vlijmen BJ, Havekes LM, van der Laarse A, van der Wall EE, Jukema JW. Tumor necrosis factor-alpha plays an important role in restenosis development. FASEB J. 2005;19:1998-2004.

31. Marfella R, Ferraraccio F, Rizzo MR, Portoghese M, Barbieri M, Basilio C, Nersita R, Siniscalchi LI, Sasso FC, Ambrosino I, Siniscalchi M, Maresca L, Sardu C, Amato G, Paolisso G, Carella C. Innate immune activity in plaque of patients with untreated and L-thyroxine-treated subclinical hypothyroidism. J Clin Endocrinol Metab. 2011;96:1015-20.

32. Grundy SM, Howard B, Smith S Jr, Eckel R, Redberg R, Bonow RO. Prevention Conference VI: Diabetes and Cardiovascular Disease: executive summary: conference proceeding for healthcare professionals from a special writing group of the American Heart Association. Circulation. 2002;105:2231-9.

33. Marfella R, Sasso FC, Siniscalchi M, Paolisso P, Rizzo MR, Ferraro F, Stabile E, Sorropago G, Calabro P, Carbonara O, Cinquegrana G, Piscione F, Ruocco A, D'Andrea D, Rapacciuolo A, Petronella P, Bresciani A, Rubino P, Mauro C, Paolisso G. Peri-procedural tight glycemic control during early percutaneous coronary intervention is associated with a lower rate of in-stent restenosis in patients with acute ST-elevation myocardial infarction. J Clin Endocrinol Metab. 2012;97:2862-71.

34. Marfella R, M DA, Di Filippo C, Siniscalchi M, Sasso FC, Ferraraccio F, Rossi $\mathrm{F}$, Paolisso $\mathrm{G}$. The possible role of the ubiquitin proteasome system in the development of atherosclerosis in diabetes. Cardiovasc Diabetol. 2007;6:35.

35. Sasso FC, Pafundi PC, Marfella R, Calabro P, Piscione F, Furbatto F, Esposito G, Galiero R, Gragnano F, Rinaldi L, Salvatore T, D'Amico M, Adinolfi LE, Sardu C. Adiponectin and insulin resistance are related to restenosis and overall new $\mathrm{PCl}$ in subjects with normal glucose tolerance: the prospective AIRE Study. Cardiovasc Diabetol. 2019;18:24.

36. Thum T, Condorelli G. Long noncoding RNAs and microRNAs in cardiovascular pathophysiology. Circ Res. 2015;116:751-62.

37. Indolfi C, laconetti C, Gareri C, Polimeni A, De Rosa S. Non-coding RNAs in vascular remodeling and restenosis. Vascul Pharmacol. 2019;114:49-63.

38. Xu F, Ahmed AS, Kang X, Hu G, Liu F, Zhang W, Zhou J. MicroRNA-15b/16 attenuates vascular neointima formation by promoting the contractile phenotype of vascular smooth muscle through targeting YAP. Arterioscler Thromb Vasc Biol. 2015;35:2145-52.

39. de Freitas RCC, Bortolin RH, Lopes MB, Tamborlin L, Meneguello L, Silbiger VN, Hirata RDC, Hirata MH, Luchessi AD, Luchessi AD. Modulation of miR$26 a-5 p$ and miR-15b-5p exosomal expression associated with clopidogrelinduced hepatotoxicity in HepG2 cells. Front Pharmacol. 2017;8:906.

40. Munoz-Pacheco P, Ortega-Hernandez A, Miana M, Cachofeiro V, FernandezCruz A, Gomez-Garre D. Ezetimibe inhibits PMA-induced monocyte/ macrophage differentiation by altering microRNA expression: a novel antiatherosclerotic mechanism. Pharmacol Res. 2012;66:536-43.

41. Richardsen E, Andersen S, Al-Saad S, Rakaee M, Nordby Y, Pedersen MI, Ness $\mathrm{N}$, Ingebriktsen LM, Fassina A, Tasken KA, Mills IG, Donnem T, Bremnes RM, Busund LT. Low expression of miR-424-3p is highly correlated with clinical failure in prostate cancer. Sci Rep. 2019;9:10662.

42. Reid G, Kirschner MB, van Zandwijk N. Circulating microRNAs: association with disease and potential use as biomarkers. Crit Rev Oncol Hematol. 2011:80:193-208.

43. Jacob NK, Cooley JV, Yee TN, Jacob J, Alder H, Wickramasinghe P, Maclean $\mathrm{KH}$, Chakravarti A. Identification of sensitive serum microRNA biomarkers for radiation biodosimetry. PLOS ONE. 2013;8:e57603.

\section{Publisher's Note}

Springer Nature remains neutral with regard to jurisdictional claims in published maps and institutional affiliations. 